\title{
PBP5, PBP6 and DacD play different roles in intrinsic $\beta$-lactam resistance of Escherichia coli
}

\author{
Correspondence \\ Anindya S. Ghosh \\ anindyain@yahoo.com
}

Received 17 October 2010

Revised 12 April 2011

Accepted 24 June 2011

\author{
Sujoy Kumar Sarkar, Mouparna Dutta, $†$ Chiranjit Chowdhury, $†$ \\ Akash Kumar and Anindya S. Ghosh
}

Department of Biotechnology, Indian Institute of Technology, Kharagpur, West Bengal PIN-721302, India

\begin{abstract}
Escherichia coli PBP5, PBP6 and DacD, encoded by dacA, dacC and dacD, respectively, share substantial amino acid identity and together constitute $\sim 50 \%$ of the total penicillin-binding proteins of E. coli. PBP5 helps maintain intrinsic $\beta$-lactam resistance within the cell. To test if PBP6 and DacD play simlar roles, we deleted dacC and dacD individually, and dacC in combination with dacA, from $E$. coli 2443 and compared $\beta$-lactam sensitivity of the mutants and the parent strain. $\beta$-Lactam resistance was complemented by wild-type, but not DD-carboxypeptidase-deficient PBP5, confirming that enzymic activity of PBP5 is essential for $\beta$-lactam resistance. Deletion of dacC and expression of PBP6 during exponential or stationary phase did not alter $\beta$-lactam resistance of a dacA mutant. Expression of DacD during mid-exponential phase partially restored $\beta$-lactam resistance of the dac $A$ mutant. Therefore, PBP5 $\mathrm{DD}$-carboxypeptidase activity is essential for intrinsic $\beta$-lactam resistance of $E$. coli and DacD can partially compensate for PBP5 in this capacity, whereas PBP6 cannot.
\end{abstract}

\section{INTRODUCTION}

Escherichia coli encodes 12 penicillin-binding proteins (PBPs), four of which (PBP4, $-5,-6$ and $\mathrm{DacD}$ ) have been reported to have DD-carboxypeptidase (DD-CPase) activity (Höltje, 1998; Denome et al., 1999; Ghosh et al., 2008). All these proteins are low molecular mass (LMM) PBPs (Ghuysen, 1991) and are dispensable for survival in vitro (Denome et al., 1999). PBP5 and PBP6 are $62 \%$ identical at the amino acid level and share 48 and $47 \%$ identity with DacD, respectively (Baquero et al., 1996). It has been suggested that these proteins might have similar physiological functions based upon their homology but only PBP5 appears to play a prominent role in maintenance of cell shape (Nelson \& Young, 2001; Nelson et al., 2002; Ghosh \& Young, 2003). PBP5, PBP6 and DacD are primarily expressed in early exponential, stationary and midexponential phases, respectively (Buchanan \& Sowell, 1982; Baquero et al., 1996; Santos et al., 2002), which may explain the different roles of these proteins in maintenance of cell shape. The number of PBP5 molecules is also two- to threefold higher than the number of PBP6 molecules in exponentially growing cells (Spratt, 1977; Dougherty et al., 1996).

It has been observed recently that loss of PBP5 makes the cells susceptible to a wide range of $\beta$-lactam antibiotics and

†These authors contributed equally to this work.

Abbreviations: DD-CPase, DD-carboxypeptidase; LMM, low molecular mass; PBP, penicillin-binding protein.

Two supplementary figures are available with the online version of this paper. this can be reversed by expression of PBP5 in trans (Sarkar et al., 2010). Similarly, deletion of some PBPs from E. coli increases susceptibility to $\beta$-lactam antibiotics (Nishimura et al., 1980; Pepper et al., 2006; Georgopapadakou, 1993). However, loss of PBPs may also increase $\beta$-lactam resistance indirectly. For example, inactivation of PBP4 triggers overproduction of the AmpC $\beta$-lactamase in Pseudomonas aeruginosa (Moya et al., 2009).

Based upon the homology of PBP5, PBP6 and DacD we hypothesized that these proteins might play similar roles in $\beta$-lactam sensitivity. To test this, we compared the $\beta$-lactam sensitivities of PBP6 and DacD mutants individually, and in the presence and absence of PBP5. Deletion of PBP6 and DacD did not change the $\beta$-lactam sensitivity of E. coli, although expression of DacD in trans could partially compensate for the loss of PBP5.

\section{METHODS}

Bacterial strains and antibiotics. Bacterial strains used in this study were derived from E. coli 2443 and are listed in Table 1. CS18$2 \mathrm{~K}$ was a gift from Professor Kevin D. Young, University of Arkansas Medical School, AR, USA. The strains were grown in Luria-Bertani (LB) broth, agar (Hi-Media), Muller-Hinton (MH) broth (Hi-Media) and M9-Glucose minimal medium, supplemented with the required amino acids (arginine, proline, leucine and threonine) and thiamine. Chloramphenicol $\left(20 \mu \mathrm{g} \mathrm{ml}^{-1}\right)$, kanamycin $\left(50 \mu \mathrm{g} \mathrm{ml}^{-1}\right)$, tetracycline $\left(25 \mu \mathrm{g} \mathrm{ml}^{-1}\right)$ and ampicillin $\left(50 \mu \mathrm{g} \mathrm{ml}^{-1}\right)$ were added where necessary. Unless otherwise specified, chemicals and reagents were purchased from Sigma. 
Table 1. E. coli strains and plasmids used in the study

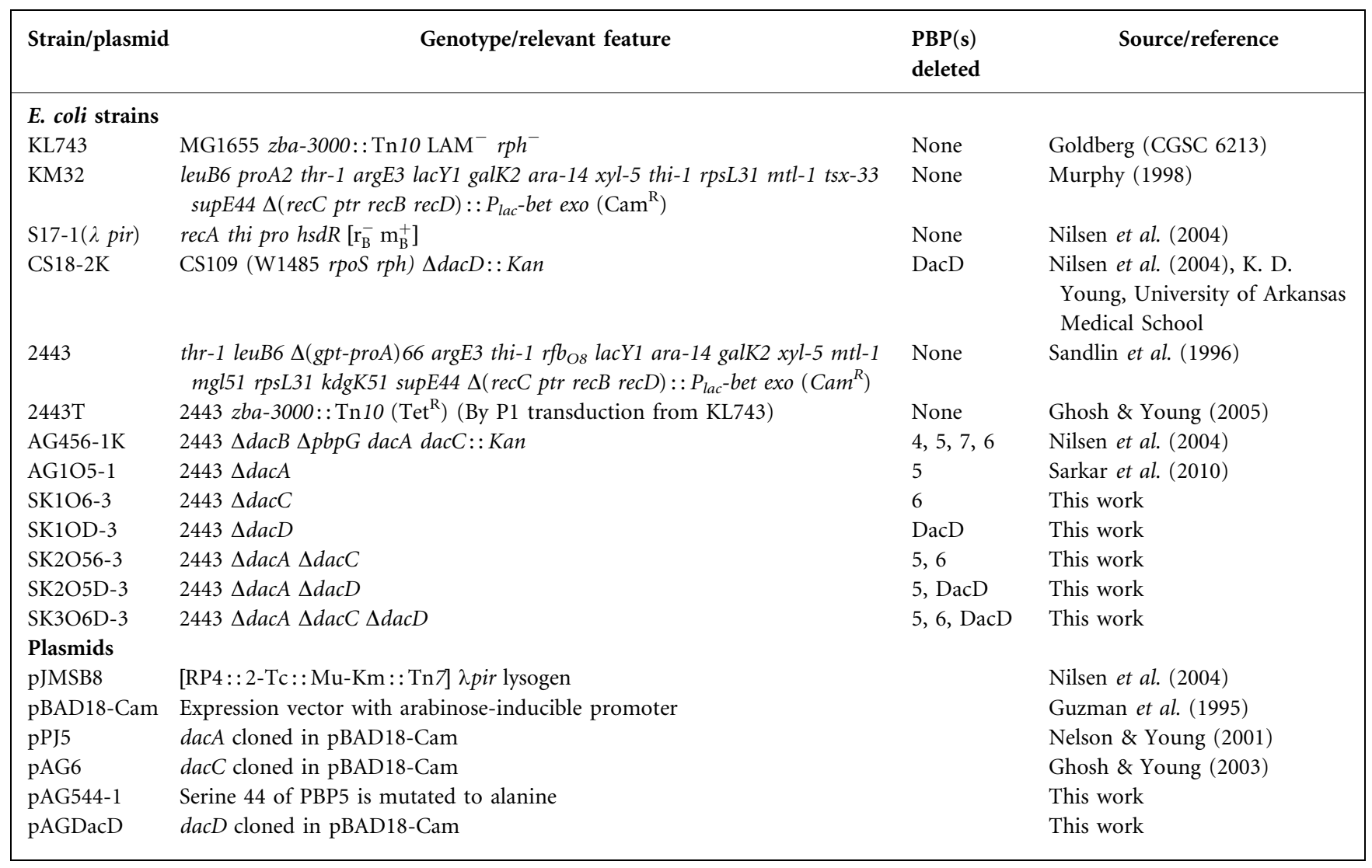

Construction of the PBP deletion mutants. PBP deletion mutants were constructed by P1 transduction from E. coli 2443T (Denome et al., 1999). E. coli $2443 \mathrm{~T}$ was previously constructed by inserting a tetracycline marker (Ghosh \& Young, 2005). The dacA mutant was constructed as described previously (Sarkar et al., 2010). The PBP6 deletion mutant E. coli 2443 (SK1O6-3) was constructed by transducing the $\Delta$ dacC: : res-npt-res $\left(\mathrm{Kan}^{\mathrm{R}}\right)$ allele from AG456-1K (Nilsen et al., 2004), and the DacD deletion mutant (SK1OD-3) was produced by transducing $\Delta d a c D$ : : res-npt-res $\left(\mathrm{Kan}^{\mathrm{R}}\right)$ allele from CS18-2K (Nilsen et al., 2004) (Table 1). Double and triple mutants were constructed from parents from which the res-npt-res cassette had been resolved (Sarkar et al., 2010) by conjugation with pJMSB8 to transiently express RP4 ParA resolvase (Sarkar et al., 2010; Nilsen et al., 2004; Kristensen et al., 1995). Kanamycin-sensitive isolates were identified by streaking on LB kanamycin agar. Deletions were confirmed by PCR and the absence of corresponding PBPs in the mutants was confirmed by labelling with Bocillin FL (Zhao et al., 1999) (Fig. 1).

Cloning. The dacD gene was amplified by PCR from E. coli 2443 genomic DNA using oligonucleotide primers 5 '-CTCTCTGCTAGCAGGAGGAATTCACCATGAAACGCCGTCTTATTATTGC-3' and 5' -CTCTCTAAGCTTTCAGGCCTTATGGTGGAAATAATCACTCAGGC- $3^{\prime}$. A Shine-Dalgarno sequence (in bold) was incorporated in the forward primer. PCR conditions were $5 \mathrm{~min}$ at $95{ }^{\circ} \mathrm{C}$, followed by 30 cycles of $95{ }^{\circ} \mathrm{C}$ for $1 \mathrm{~min}, 60{ }^{\circ} \mathrm{C}$ for $1 \mathrm{~min}$ and $72{ }^{\circ} \mathrm{C}$ for $1 \mathrm{~min}$. The PCR product was digested with NheI and HindIII (underlined in the primer sequence) and ligated into the multiple cloning site of pBAD18Cam. Transformants were selected on chloramphenicol plates and positive clones were confirmed by DNA sequencing (MWG Biotech).

Site-directed mutagenesis of PBP5. The active-site serine ( $\left.\mathrm{Ser}_{44}\right)$ of PBP5 was replaced with alanine (AGC to GCC) through site-directed mutagenesis of the parent plasmid pPJ5, using a QuikChange mutagenesis kit (Stratagene) and oligonucleotide primers 5'-CGCCGCGATCCTGCCGCCCTGACCAAAATGATG-3' and 5'CATCATTTTGGTCAGGGCGGCAGGATCGCGGCG-3'， according to the manufacturer's instructions to produce plasmid pAG544-1 (bold/italic type indicates changed nucleotides).

MIC testing. MICs were determined by a micro-broth dilution method following CLSI guidelines (CLSI, 2007) and as we have described previously (Sarkar et al., 2010; Sarkar \& Ghosh, 2008) in 96-well plates in a total volume of $300 \mu \mathrm{l}$ per well. Wells were inoculated with $\sim 10^{5}$ c.f.u. per well, and antibiotics were tested at concentrations from 1 to $0.006 \mu \mathrm{g} \mathrm{ml}^{-1}$ by twofold step dilution (Stubbs et al., 2007). The plates were incubated for $18 \mathrm{~h}$ at $37^{\circ} \mathrm{C}$ and growth of the cultures was monitored by measuring $\mathrm{OD}_{600}$ using a Multiskan Spectrum-1500 Spectrophotometer (Thermo Scientific). MIC tests were performed in triplicate and were repeated at least six times.

Complementation experiments. MICs for SK1O6-3, SK1OD-3, SK2O56-3, SK2O5D-3 and the triple mutant SK3O6D-3 were determined after complementation with plasmids encoding PBP5 (pPJ5), PBP6 (pAG6), DacD (pAGDacD) and mutated PBP5 (pAG544-1), all of which were constructed in the pBAD18-Cam backbone (see Table 1). Prior to MIC assays, cells were grown in the presence of chloramphenicol and $0.2 \%$ glucose to prevent expression from the pBAD promoter. The cells were then washed twice in $\mathrm{MH}$ Broth, resuspended in $\mathrm{MH}$ broth and treated with a varying amounts of arabinose $(0.0005-0.2 \% \mathrm{w} / \mathrm{v})$ to induce PBP expression from the plasmids (Fig. 1).

Growth kinetics and time-kill assays. These assays were performed as described previously, with minor modifications (Kumar et al., 


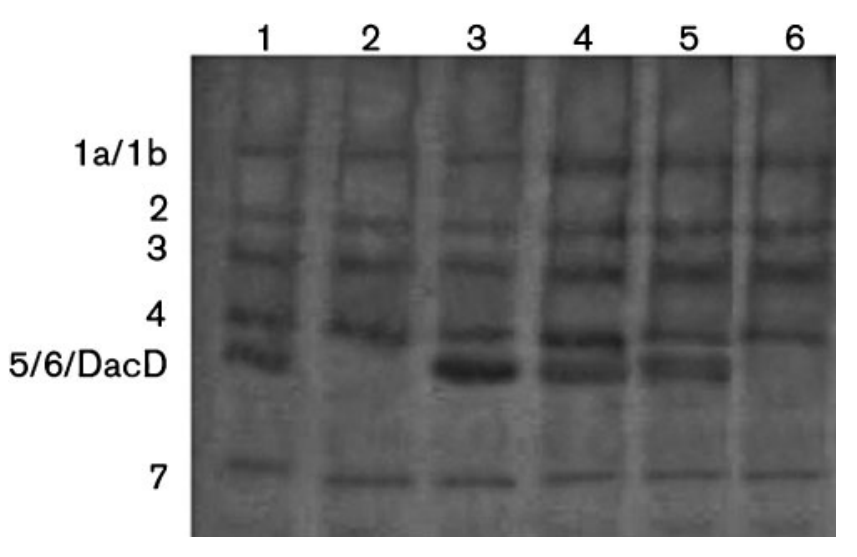

Fig. 1. Labelling of PBP5 with fluorescent penicillin. Isolated membranes (protein content $\sim 300 \mu \mathrm{g}$ ) were labelled with Bocillin $\mathrm{FL}(50 \mu \mathrm{M})$ at $30{ }^{\circ} \mathrm{C}$ for $30 \mathrm{~min}$, solubilized with $1 \%$ Sarkosyl and analysed by using $12 \%$ SDS-PAGE (100 $\mu$ g protein per lane). Lanes: 1, E. coli 2443; 2, SK2O56-3; 3, SK2O56-3 expressing PBP5 from the arabinose promoter in the presence of an optimal concentration of arabinose $(0.0005 \%)$ at exponential phase; 4, SK2O56-3 expressing PBP6 from the arabinose promoter with arabinose $(0.2 \%)$ at stationary phase; 5 , SK2O56-3 expressing DacD from the arabinose promoter with arabinose $(0.001 \%)$ at mid-exponential phase; 6, SK2O56-3 complemented with pAG544-1 with arabinose (0.2\%).

2008; MacLeod et al., 2009). Briefly, cells $\left(10^{5}\right.$ c.f.u. $\left.\mathrm{ml}^{-1}\right)$ were inoculated into $\mathrm{MH}$ broth containing ampicillin or cefadroxil (representative antibiotics from penicillin and cephalosporin groups, respectively). The cultures were incubated at $37^{\circ} \mathrm{C}$ and viable cells were enumerated by plating on $\mathrm{LB}$ and $\mathrm{MH}$ agar.

\section{RESULTS}

$\beta$-Lactams are substrate analogues of the D-alanyl-Dalanine moiety of muropeptides (Park \& Strominger, 1957), and kill bacteria by inactivation of high molecular mass PBPs. Inactivation or alteration of the affinities of some HMM PBPs for their substrates can impact sensitivity to $\beta$-lactams (Gotoh et al., 1990; Gerrits et al., 2002, 2006; Ghosh et al., 1998). Loss of PBP5 increases E. coli $\beta$-lactam sensitivity by four- to eightfold, indicating that LMM PBPs contribute to this phenomenon (Sarkar et al., 2010). PBP6 and DacD share structural similarities with PBP5 so we speculated that these proteins might play similar roles in $\beta$-lactam sensitivity.

\section{Loss of PBP6 or DacD alone or in a PBP5 mutant background does not affect $\beta$-lactam sensitivity}

To test if PBP6 or DacD contribute to intrinsic $\beta$-lactam resistance, we compared the $\beta$-lactam sensitivities of PBP5, PBP6 and DacD single and multiple mutant strains. PBP5 mutants had four- to eightfold higher sensitivity to $\beta$-lactams, consistent with our previous findings (Sarkar et al., 2010). $\beta$-Lactam resistance of PBP5 mutants was restored by the expression of wild-type, but not a DD-CPase deficient PBP5 (Ser44 to Ala44), in trans. In contrast, the phenotypes of single PBP6 and DacD mutant strains were indistinguishable from wild-type (Table 2). To test if PBP5 was dominant over and masked contributions of PBP6 and/or DacD, PBP6 and DacD were deleted singly, and in combination, from the PBP5 mutant and the $\beta$-lactam resistance phenotypes of these strains were compared. PBP5, PBP5/6 and PBP5/DacD and PBP5/6/DacD mutants yielded almost identical results. These results indicate that DD-CPase activity of PBP5, but not of PBP6 or DacD, is critical to the intrinsic $\beta$-lactam resistance of $E$. coli.

\section{Overexpression of DacD, but not PBP6, partially restores $\beta$-lactam sensitivity of PBP5 mutants}

DacD and PBP6 are expressed at lower copy numbers than PBP5. This suggests that these proteins might not have been expressed at sufficient levels to affect $\beta$-lactam resistance. To test this, we overexpressed PBP5, DacD and PBP6 from inducible multi-copy plasmids in PBP5 mutant strains and assessed $\beta$-lactam resistance. PBP5 restored $\beta$-lactam resistance of PBP5/6 and PBP5/DacD mutants, whereas overexpression of PBP6 had no effect. DacD partially (25$50 \%$ ) restored $\beta$-lactam resistance of PBP5/6 and PBP5/6/ DacD mutants. These results suggest PBP5 and DacD might have related roles in $\beta$-lactam resistance.

\section{Expression of PBP6 during stationary phase does not restore $\beta$-lactam resistance of PBP5 mutants}

PBP5, PBP6 and DacD expression are maximal at different points in E. coli development (Ghosh et al., 2008). For example, PBP6 expression increases in stationary phase (Santos et al., 2002) whereas PBP5 levels peak during exponential growth. Thus, we tested if expressing these PBPs in different growth phases affected $\beta$-lactam resistance (Fig. 2). PBP expression from plasmids was induced by adding arabinose to the cultures in early exponential, mid-exponential or stationary phase. Subinhibitory levels of ampicillin $\left(0.25 \mu \mathrm{g} \mathrm{ml}^{-1}\right)$ or cefadroxil $\left(2 \mu \mathrm{g} \mathrm{ml}^{-1}\right)$ were used to assess growth inhibition. Expression of PBP5 in trans in early exponential phase completely restored $\beta$-lactam resistance of PBP5 mutants. DacD partially restored $\beta$-lactam resistance when it was expressed in mid-exponential phase. Expression of PBP6 in any growth phase failed to affect $\beta$-lactam resistance. These results suggest that PBP5 and DacD mediate $\beta$-lactam resistance of E. coli in different growth phases.

\section{Different $\beta$-lactam resistance phenotypes of PBP mutants are not related to growth rate}

Growth rate is an important determinant of $\beta$-lactam sensitivity because these compounds primarily affect cells during exponential phase (Anwar et al., 1990). We suspected that minor variations in growth rate of different LMM PBP mutants might be reflected by increased 
Table 2. $\beta$-Lactam sensitivities (MIC) of E. coli 2443 and the PBP mutants

All concentrations are in $\mu \mathrm{g} \mathrm{ml}^{-1}$.

\begin{tabular}{|c|c|c|c|c|c|c|c|c|}
\hline E. coli strain & Amoxicillin & Piperacillin & Ampicillin & Penicillin G & Cefadroxil & Cefalexin & Cefalothin & Cefaclor \\
\hline AG1O5-1 & 1 & 0.25 & 0.5 & 32 & 4 & 2 & 2 & 0.5 \\
\hline SK1O6-3 & 4 & 1 & 2 & 125 & 16 & 8 & 16 & 4 \\
\hline SK1OD-3 & 4 & 1 & 2 & 125 & 16 & 8 & 16 & 4 \\
\hline SK1O6-3 + PBP6 & 4 & 1 & 2 & 125 & 16 & 8 & 16 & 4 \\
\hline SK2O56-3 + PBP5 & 4 & 1 & 2 & 125 & 16 & 8 & 16 & 4 \\
\hline SK2O56-3 + PBP6 & 1 & 0.25 & 0.5 & 32 & 4 & 2 & 2 & 0.5 \\
\hline SK2O56-3/pAG544-1 & 1 & 0.25 & 0.5 & 32 & 4 & 2 & 2 & 0.5 \\
\hline SK2O56-3+DacD & 2 & 0.5 & 1 & 64 & 8 & 4 & 4 & 2 \\
\hline SK2O5D-3 & 1 & 0.25 & 0.5 & 32 & 4 & 2 & 2 & 0.5 \\
\hline SK2O5D-3+DacD & 2 & 0.5 & 1 & 64 & 8 & 4 & 4 & 2 \\
\hline
\end{tabular}

sensitivity to subinhibitory concentrations of $\beta$-lactams. To test this, we compared the growth rates of LMM PBP mutant strains in the presence of subinhibitory concentrations (half, quarter and eighth of the MIC) of ampicillin and cefadroxil. Growth inhibition of all of the LMM PBP mutants was proportional to the concentration of antibiotics and was not affected by genotype (Supplementary Fig. S1, available with the online version of this paper). Similar growth rates in all the mutants indicated that differences in intrinsic $\beta$-lactam resistance of PBP5, PBP6 and DacD mutants are not related to growth rate.

\section{Analysis of killing kinetics indicate that PBP5, PBP6 and DacD have different roles in intrinsic $\beta$-lactam resistance}

MIC assays can obscure differences in the speed of killing by antibiotics, so we performed killing kinetics assays using a range of antibiotic concentrations near the MIC of the parent strain to test whether LMM PBP mutants had different phenotypes. Ampicillin and cefadroxil were chosen as representatives for penicillins and cephalosporins in these assays (see Methods and Kumar et al., 2008 and MacLeod et al., 2009 for details). The general patterns observed in the experiments above were duplicated in these assays. Briefly, expression of PBP5 fully and DacD partially complemented $\beta$-lactam resistance in PBP5 mutants whereas PBP6 had no effect (Supplementary Fig. S2, available with the online version of this paper).

\section{DISCUSSION}

We showed that loss of PBP6 and DacD from wild-type E. coli does not affect the $\beta$-lactam sensitivity of PBP5 mutants and that expression of PBP5 in trans fully restores wild-type $\beta$-lactam resistance to a PBP5/6/DacD triple
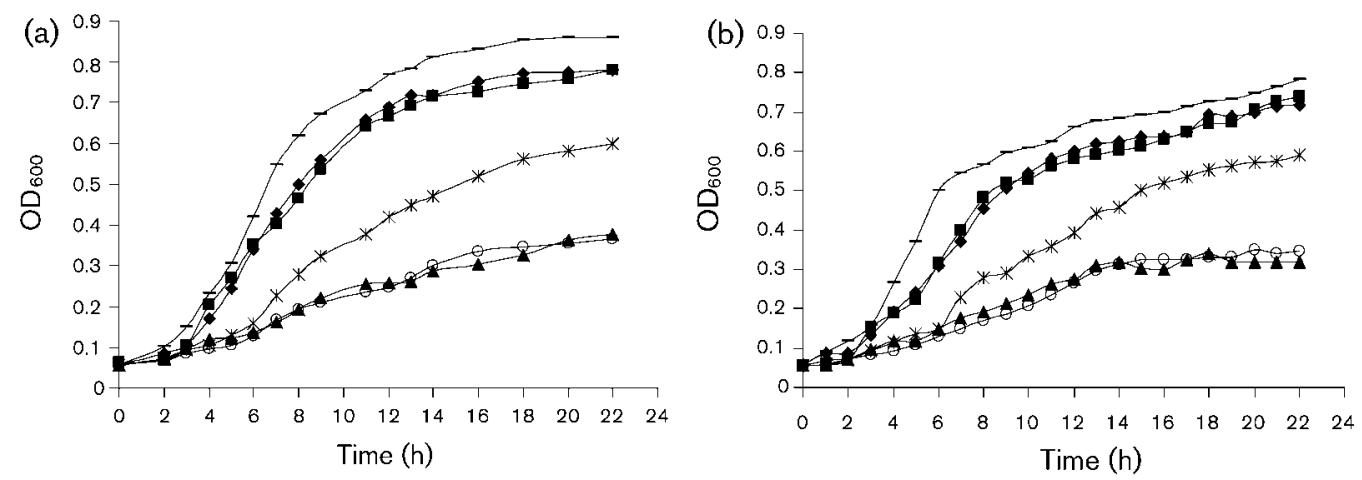

Fig. 2. Phase expression studies in the presence of (a) ampicillin $\left(0.25 \mu \mathrm{g} \mathrm{ml}^{-1}\right)$ and (b) cefadroxil $\left(2 \mu \mathrm{g} \mathrm{ml}^{-1}\right)$. The strains are E. coli 2443 (in the absence of antibiotic; -), E. coli $2443(\diamond)$, SK2O56-3 ( $\bigcirc)$, SK2O56-3 expressing PBP5 ( $\mathbf{\square})$, SK2O56-3 expressing PBP6 $(\boldsymbol{\Delta})$ and SK2O56-3 expressing DacD (*). 
mutant strain. $\beta$-Lactam sensitivity of PBP5 mutants is related to PBP5 DD-CPase activity because catalytically inactive PBP5 did not complement this mutation. Although expression of PBP6 in any growth phase failed to restore resistance to a PBP5 mutant, expression of DacD during mid-exponential phase partially restored $\beta$-lactam resistance. Collectively, these results indicate that PBP5 mediates intrinsic $\beta$-lactam resistance in wild-type $E$. coli whereas PBP6 and DacD do not. Separately, the results imply that the cellular functions of DacD and PBP5 may be more closely related to one another than they are to PBP6.

A few hypotheses could explain the different roles of PBP5 and PBP6. One possibility is that PBP5 and PBP6 have similar enzymic functions but that loss of PBP6 fails to effect $\beta$-lactam resistance because the protein is not present when $\beta$-lactam antibiotics act against the cells. Consistent with this, PBP6 accumulates in stationary phase cells whereas expression of PBP5 is maximal in exponential phase cells. However, overexpression of PBP6 in exponential phase cells did not affect $\beta$-lactam resistance. Thus, if PBP5 maintains resistance by 'trapping' $\beta$-lactam antibiotics, as we have previously proposed (Sarkar et al., 2010), PBP6 does not seem to be able to perform this function, regardless of when it is expressed. A caveat is that if other co-factors that modulate PBP6 activity are also expressed in stationary phase, PBP6 expressed during exponential phase might not be fully active. Biochemical and in silico analyses suggest other explanations for the different roles of PBP5 and PBP6. PBP5 and PBP6 both bind $\beta$-lactams efficiently. The acylation rate $\left(k_{2} / K\right)$ of PBP6 for $\beta$-lactams and some small DD-CPase substrates, such as $\mathrm{Ac}_{2}$-L-Lys-D-Ala-D-Ala, is actually slightly higher than that of PBP5. However, PBP6 cannot bind the peptidoglycan mimetic pentapeptide substrate (Chowdhury et al., 2011). This has been linked to differences in the microarchitecture of the active sites of these proteins (Chowdhury \& Ghosh, 2011). Also, PBP5 deacylates $\beta$-lactam (Bocillin FL) more rapidly than does PBP6 (Chowdhury et al., 2010). Thus, the effective pool of PBP5 available to bind $\beta$-lactams could be larger than that of PBP6 even if their protein copy numbers in cells are similar.

DacD partially rescued $\beta$-lactam resistance of PBP5 mutants, suggesting that these proteins have related functions in vivo. Baquero et al. (1996) previously proposed that DacD compensates for other DD-CPases in their absence and our observations support this interpretation. DacD can utilize pentapeptide muropeptides and maintained $5 \%$ of the residual DD-CPase activity of an $E$. coli strain from which all of the other DD-CPases had been deleted (Baquero et al., 1996). That $5 \%$ might be sufficient to impart normal physiological function (Waxman \& Strominger, 1983). Unfortunately, little is known about the structure, substrate specificity and/or kinetics of this enzyme at present (Nelson et al. 2002; Ghosh et al. 2008). We hope to address these questions in the future because DacD homologues are present in a wide variety of pathogenic Gram-negative bacteria and our data suggest that these proteins could play unappreciated roles in $\beta$-lactam resistance.

\section{ACKNOWLEDGEMENTS}

The authors are deeply indebted to David E. Nelson for editing of the manuscript. This work has been supported in parts by research grants from the Indian Council for Medical Research (ICMR) Department of Biotechnology (DBT), Government of India, to A. S. G. M. D. was supported by a grant from the Council of Scientific and Industrial Research (CSIR) to A. S. G.

\section{REFERENCES}

Anwar, H., Dasgupta, M. K. \& Costerton, J. W. (1990). Testing the susceptibility of bacteria in biofilms to antibacterial agents. Antimicrob Agents Chemother 34, 2043-2046.

Baquero, M. R., Bouzon, M., Quintela, J. C., Ayala, J. A. \& Moreno, F. (1996). dacD, an Escherichia coli gene encoding a novel penicillinbinding protein (PBP6b) with DD-carboxypeptidase activity. J Bacteriol 178, 7106-7111.

Buchanan, C. E. \& Sowell, M. O. (1982). Synthesis of penicillinbinding protein 6 by stationary-phase Escherichia coli. J Bacteriol 151, 491-494.

Chowdhury, C. \& Ghosh, A. S. (2011). Differences in active-site microarchitecture explain the dissimilar behaviors of PBP5 and 6 in Escherichia coli. J Mol Graph Model 29, 650-656.

Chowdhury, C., Nayak, T. R., Young, K. D. \& Ghosh, A. S. (2010). A weak DD-carboxypeptidase activity explains the inability of PBP6 to substitute for PBP5 in maintaining normal cell shape in Escherichia coli. FEMS Microbiol Lett 303, 76-83.

CLSI (2007). Performance standards for antimicrobial susceptibility testing. Seventeenth informational supplement. Document M100-S17. CLSI. Wayne, PA: Clinical and Laboratory Standards Institute.

Denome, S. A., Elf, P. K., Henderson, T. A., Nelson, D. E. \& Young, K. D. (1999). Escherichia coli mutants lacking all possible combinations of eight penicillin binding proteins: viability, characteristics, and implications for peptidoglycan synthesis. J Bacteriol 181, 3981-3993.

Dougherty, T. J., Kennedy, K., Kessler, R. E. \& Pucci, M. J. (1996). Direct quantitation of the number of individual penicillin-binding proteins per cell in Escherichia coli. J Bacteriol 178, 6110-6115.

Georgopapadakou, N. H. (1993). Penicillin-binding proteins and bacterial resistance to $\beta$-lactams. Antimicrob Agents Chemother 37, 2045-2053.

Gerrits, M. M., Schuijffel, D., van Zwet, A. A., Kuipers, E. J., Vandenbroucke-Grauls, C. M. \& Kusters, J. G. (2002). Alterations in penicillin-binding protein $1 \mathrm{~A}$ confer resistance to $\beta$-lactam antibiotics in Helicobacter pylori. Antimicrob Agents Chemother 46, 2229-2233.

Gerrits, M. M., Godoy, A. P., Kuipers, E. J., Ribeiro, M. L., Stoof, J., Mendonça, S., van Vliet, A. H., Pedrazzoli, J., Jr \& Kusters, J. G. (2006). Multiple mutations in or adjacent to the conserved penicillinbinding protein motifs of the penicillin-binding protein $1 \mathrm{~A}$ confer amoxicillin resistance to Helicobacter pylori. Helicobacter 11, 181-187.

Ghosh, A. S. \& Young, K. D. (2003). Sequences near the active site in chimeric penicillin binding proteins 5 and 6 affect uniform morphology of Escherichia coli. J Bacteriol 185, 2178-2186.

Ghosh, A. S., Kar, A. K. \& Kundu, M. (1998). Alterations in high molecular mass penicillin-binding protein 1 associated with $\beta$-lactam 
resistance in Shigella dysenteriae. Biochem Biophys Res Commun 248, 669-672.

Ghosh, A. S. \& Young, K. D. (2005). Helical disposition of proteins and lipopolysaccharide in the outer membrane of Escherichia coli. J Bacteriol 187, 1913-1922.

Ghosh, A. S., Chowdhury, C. \& Nelson, D. E. (2008). Physiological functions of D-alanine carboxypeptidases in Escherichia coli. Trends Microbiol 16, 309-317.

Ghuysen, J. M. (1991). Serine $\beta$-lactamases and penicillin-binding proteins. Annu Rev Microbiol 45, 37-67.

Gotoh, N., Nunomura, K. \& Nishino, T. (1990). Resistance of Pseudomonas aeruginosa to cefsulodin: modification of penicillinbinding protein 3 and mapping of its chromosomal gene. J Antimicrob Chemother 25, 513-523.

Guzman, L. M., Belin, D., Carson, M. J. \& Beckwith, J. (1995). Tight regulation, modulation, and high-level expression by vectors containing the arabinose $\mathrm{P}_{\mathrm{BAD}}$ promoter. J Bacteriol 177, 4121-4130.

Höltje, J. V. (1998). Growth of the stress-bearing and shapemaintaining murein sacculus of Escherichia coli. Microbiol Mol Biol Rev 62, 181-203.

Kristensen, C. S., Eberl, L., Sanchez-Romero, J. M., Givskov, M., Molin, S. \& De Lorenzo, V. (1995). Site-specific deletions of chromosomally located DNA segments with the multimer resolution system of broad-host-range plasmid RP4. J Bacteriol 177, 52-58.

Kumar, A., Khan, I. A., Koul, S., Koul, J. L., Taneja, S. C., Ali, I., Ali, F., Sharma, S., Mirza, Z. M. \& other authors (2008). Novel structural analogues of piperine as inhibitors of the NorA efflux pump of Staphylococcus aureus. J Antimicrob Chemother 61, 1270-1276.

MacLeod, D. L., Barker, L. M., Sutherland, J. L., Moss, S. C., Gurgel, J. L., Kenney, T. F., Burns, J. L. \& Baker, W. R. (2009). Antibacterial activities of a fosfomycin/tobramycin combination: a novel inhaled antibiotic for bronchiectasis. J Antimicrob Chemother 64, 829-836.

Moya, B., Dötsch, A., Juan, C., Blázquez, J., Zamorano, L., Haussler, S. \& Oliver, A. (2009). $\beta$-Lactam resistance response triggered by inactivation of a nonessential penicillin-binding protein. PLoS Pathog 5, e1000353.

Murphy, K. C. (1998). Use of recombinant $\lambda$ recombination function to promote gene replacement in Escherichia coli. J Bacteriol 180, 20632071.

Nelson, D. E. \& Young, K. D. (2001). Contributions of PBP5 and DDcarboxypeptidase penicillin binding proteins to maintenance of cell shape in Escherichia coli. J Bacteriol 183, 3055-3064.
Nelson, D. E., Ghosh, A. S., Paulson, A. L. \& Young, K. D. (2002). Contribution of membrane-binding and enzymatic domains of penicillin binding protein 5 to maintenance of uniform cellular morphology of Escherichia coli. J Bacteriol 184, 3630-3639.

Nilsen, T., Ghosh, A. S., Goldberg, M. B. \& Young, K. D. (2004). Branching sites and morphological abnormalities behave as ectopic poles in shape-defective Escherichia coli. Mol Microbiol 52, 1045-1054.

Nishimura, Y., Suzuki, H., Hirota, Y. \& Park, J. T. (1980). A mutant of Escherichia coli defective in penicillin-binding protein 5 and lacking D-alanine carboxypeptidase IA. J Bacteriol 143, 531-534.

Park, J. T. \& Strominger, J. L. (1957). Mode of action of penicillin. Science 125, 99-101.

Pepper, E. D., Farrell, M. J. \& Finkel, S. E. (2006). Role of penicillinbinding protein $1 \mathrm{~b}$ in competitive stationary-phase survival of Escherichia coli. FEMS Microbiol Lett 263, 61-67.

Sandlin, R. C., Goldberg, M. B. \& Maurelli, A. T. (1996). Effect of Oside chain length and composition on the virulence of Shigella flexneri 2a. Mol Microbiol 22, 63-73.

Santos, J. M., Lobo, M., Matos, A. P., De Pedro, M. A. \& Arraiano, C. M. (2002). The gene bolA regulates dacA (PBP5), dacC (PBP6) and ampC (AmpC), promoting normal morphology in Escherichia coli. Mol Microbiol 45, 1729-1740.

Sarkar, S. K. \& Ghosh, A. S. (2008). Involvement of O8-antigen in altering $\beta$-lactam antibiotic susceptibilities in Escherichia coli. FEMS Microbiol Lett 282, 59-64.

Sarkar, S. K., Chowdhury, C. \& Ghosh, A. S. (2010). Deletion of penicillin-binding protein 5 (PBP5) sensitises Escherichia coli cells to $\beta$-lactam agents. Int J Antimicrob Agents 35, 244-249.

Spratt, B. G. (1977). Properties of the penicillin-binding proteins of Escherichia coli K12. Eur J Biochem 72, 341-352.

Stubbs, K. A., Balcewich, M., Mark, B. L. \& Vocadlo, D. J. (2007). Small molecule inhibitors of a glycoside hydrolase attenuate inducible AmpC-mediated beta-lactam resistance. J Biol Chem 282, 2138221391.

Waxman, D. J. \& Strominger, J. L. (1983). Penicillin-binding proteins and the mechanism of action of $\beta$-lactam antibiotics. Annu Rev Biochem 52, 825-869.

Zhao, G., Meier, T. I., Kahl, S. D., Gee, K. R. \& Blaszczak, L. C. (1999). BOCILLIN FL, a sensitive and commercially available reagent for detection of penicillin-binding proteins. Antimicrob Agents Chemother 43, 1124-1128.

Edited by: U. Dobrindt 